\title{
Características de los factores de riesgo cardiovascular en una población urbana y rural de la selva peruana, julio - 2014
}

Yordan Martínez-Espichán ${ }^{1, a}$, Rommel Zambrano-Huailla, ${ }^{1,2, a}$, Alexander Zambrano-Huailla ${ }^{1,2, a}$, Anibal Manrique-Acha ${ }^{1, a}$, Jean Carlo Mayta-Calderón ${ }^{1, a}$, Alejandra Cardenas-Rojas ${ }^{1, a}$, Julissa Mogollón-Lavi, a, Esthefany Seminario-Azula1,a, Stefano Giannoni-Luza',a, Gabriela Gonzales-Pariona ${ }^{1, a}$, Mitchelle Linares-Delgado ${ }^{1, a}$

\section{RESUMEN}

Objetivo: Determinar las características del lugar de residencia y los factores de riesgo cardiovascular en una población de la selva peruana en julio 2014.

Materiales y métodos: Estudio descriptivo transversal realizado en individuos entre 30 y 74 años sin diagnóstico ni tratamiento de enfermedad cardiovascular en una población urbana y una rural del distrito de Yantaló. La muestra se obtuvo a partir del programa Power Analysis and Sample Size Software (PASS) y estuvo conformada por 268 personas (152 habitantes urbanos y 116 rurales). Los factores estudiados fueron hipertensión arterial (HTA), diabetes mellitus (DM), índice de masa corporal (IMC), índice cintura-cadera (ICC) y tabaquismo.

Resultados: La población urbana presentó un mayor porcentaje de HTA $(18,4 \%)$, mientras que los otros factores de riesgo no presentaron diferencias significativas.

Conclusiones: El único factor de riesgo que mostró asociación significativa con el lugar de residencia fue la HTA. En contraste, los otros factores de riesgo del estudio no presentaron diferencias entre ambas poblaciones, lo que demostraría que el distrito de Yantaló se encuentra inmerso en un proceso de transición epidemiológica debido a la urbanización.

Palabras clave: Hipertensión; diabetes mellitus; índice de masa corporal; población urbana; población rural; factor de riesgo.

\section{Characteristics of cardiovascular risk factors in an urban and rural population of the Peruvian jungle - July, 2014}

ABSTRACT

Objective: To determine the characteristics of the place of residence and the cardiovascular risk factors in a Peruvian jungle population in July 2014.

Materials and methods: A descriptive cross-sectional study conducted in individuals between 30 and 74 years old without a diagnosis of or treatment for a cardiovascular disease in an urban and rural population of the district of Yantaló. The sample was obtained using the Power Analysis and Sample Size Software (PASS) program, and consisted of 268 people (152 urban and 116 rural residents). The studied factors were hypertension (HBP), diabetes mellitus (DM), body mass index (BMI), waist-hip ratio (WHR) and smoking.

Results: The urban population had higher rates of hypertension (18.4\%), while the other risk factors showed no significant differences between the two populations.

Conclusions: The only risk factor that showed a significant association with the place of residence was hypertension. In contrast, the other risk factors of the study showed no differences between the two populations, which demonstrates that the district of Yantalo is involved in a process of epidemiological transition due to urbanization.

Keywords: Hypertension; diabetes mellitus; body mass index; urban population: rural population: risk factors.

1. Estudiante de Medicina Humana.

2. Sociedad Científica de Estudiantes de Medicina.

a. Facultad de Medicina Humana, Universidad de San Martín de Porres. Lima, Perú. 
Yordan Martínez-Espichán, Rommel Zambrano-Huailla, Alexander Zambrano-Huailla, Anibal Manrique-Acha, Jean Carlo Mayta-Calderón, Alejandra Cardenas-Rojas, Julissa Mogollón-Lavi, Esthefany Seminario-Azula, Stefano Giannoni-Luza, Gabriela Gonzales-Pariona, Mitchelle Linares-Delgado

\section{INTRODUCCIÓN}

Las enfermedades cardiovasculares (ECV) representan una de las principales causas de muerte y discapacidad a nivel mundial ${ }^{(1)}$. Sólo en el 2012, produjeron el $31 \%$ del total de muertes alrededor del mundo, provocando mayor impacto en países de medianos y bajos ingresos ${ }^{(2)}$. Existen factores de riesgo biológicos y comportamentales para desarrollar ECV, que controlados y prevenidos mediante detección precoz y tratamiento temprano, tendrían un efecto favorable en la economía de los sectores menos favorecidos $^{(2)}$.

En el Perú, estudios nos revelan que estos factores de riesgo incrementan con la edad ${ }^{(1,3)}$, además, varían de una población a otra, dependiendo esto de los caracteres sociales, económicos y culturales ${ }^{(3)}$.

El presente estudio tuvo como objetivo determinar la asociación entre el lugar de residencia y los factores de riesgo cardiovasculares en una población de la selva peruana en julio 2014.

\section{MATERIALES Y MÉTODOS}

Se usó un diseño descriptivo transversal prospectivo y la ejecución del estudio fue en julio de 2014 en el distrito de Yantaló, región San Martín.

Yantaló, uno de los distritos menos poblados de la provincia de Moyobamba (3310 habitantes) se halla a $875 \mathrm{msnm}$, con una temperatura promedio de $22^{\circ} \mathrm{C}$ y un nivel socioeconómico de pobre y no pobre ${ }^{(4,5)}$. El grupo investigador accedió a la Fundación Yantaló para realizar un estudio de comparación de riesgo cardiovascular en la selva, debido a que no se cuenta con información suficiente sobre este problema en este tipo de poblaciones.

Para la muestra se utilizó el programa Power Analysis and Sample Size Software (PASS), un programa de computadora que sirve para la estimación de tamaño de muestra o la determinación de la potencia de una prueba estadística o intervalo de confianza, obteniéndose 152 personas para el área urbana y 116 personas para el área rural. Para la población urbana se utilizó el muestreo por conglomerados, donde primero se obtuvo el mapa completo del área urbana de Yantaló, entregado por la municipalidad del distrito, y luego se sortearon el total de manzanas encontradas dentro de los límites de la zona urbana, las cuales fueron encuestadas según el orden en el cual salían en el sorteo hasta llegar a la muestra representativa; mientras que en la población rural se empleó el muestreo incidental, donde deliberadamente se seleccionaron a las personas residentes del área rural. Se siguieron las definiciones, de población urbana y rural, establecidas por el Instituto Nacional de Estadística e Informática del Perú ${ }^{(6)}$.

Se incluyó en el estudio a toda persona, hombre o mujer no gestante, con previa firma del consentimiento informado, de 30 a 74 años de edad, sin diagnóstico ni tratamiento de ECV y residente por más de un año en el lugar de estudio. El rango de edad de la población se seleccionó basado en el estudio de Framingham ${ }^{(7)}$.
La recolección de los datos se realizó en 21 días. La primera fase incluyó una encuesta, desarrollada por los autores, recolectándose lo siguiente: datos sociodemográficos, antecedentes personales y/o familiares de ECV y/o Diabetes Mellitus (DM) diagnosticados por algún médico y determinación de tabaquismo.

La segunda fase del estudio se completó en el Centro de Salud de Yantaló. Los autores fueron capacitados previamente para la recolección de los datos, que incluyó: diagnóstico de Hipertensión Arterial (HTA) mediante el promedio de al menos 2 tomas de presión arterial separadas por 5 minutos en posición sedente y en reposo ${ }^{(8)}$, se utilizaron tensiómetros digitales automatizados (marca CITIZEN, modelo $\mathrm{CH}-452$ ), su uso está recomendado en lugar del esfigmomanómetro convencional para evitar el sesgo de medición, limitante del propósito del estudio ${ }^{(9)}$; determinación de la talla mediante un tallímetro que sigue las recomendaciones del Centro Nacional de Alimentación y Nutrición (CENAN); determinación del peso mediante una balanza digital electrónica calibrada (Seca Alpha, GmbH \& Co., Igni, France; rango 0,1-150 kg, precisión $100 \mathrm{~g}$; determinación del índice cintura/cadera (ICC), la medición de la cintura se realizó tomando el punto medio entre la arcada costal inferior y cresta iliaca superior a nivel de la línea axilar anterior, y la cadera, en la parte más ancha al nivel bitrocantéreo; el índice de masa corporal (IMC) y el ICC fueron analizadas según lo establecido por la Organización Mundial de la Salud (OMS) ${ }^{(10,11)}$; y la determinación del consumo de tabaco, según lo establecido por la Guía Nacional de Abordaje Técnico al Tabaquismo ${ }^{(12)}$; se tipificó al fumador en activo y pasivo.

Para el análisis de los datos se empleó el programa GraphPad Prism Versión 5.01. Los resultados se expresaron como porcentajes y promedios. La validación estadística se realizó mediante las pruebas de Chi-cuadrado y la prueba exacta de Fisher.

El presente estudio y el consentimiento informado fueron aprobados por el Comité Institucional de Ética en Investigación de la Universidad de San Martín de Porres y la Fundación Yantaló.

\section{RESULTADOS}

La población de estudio fue de 268 personas en total. En la zona urbana se obtuvieron datos de 62 hombres $(40,8 \%)$ y 90 mujeres $(59,2 \%)$ y en la zona rural de 53 hombres $(45,7 \%)$ y 63 mujeres $(54,3 \%)$.

Los hombres del área rural presentaron un valor promedio mayor de IMC $(25,24 \pm 3,47)$ que los del área urbana $(25,90 \pm$ $2,74)$; en contraste, las mujeres del área urbana mostraron un promedio mayor de IMC $(26,95 \pm 4,1)$ comparado con las del área rural $(26,93 \pm 4,1)$. Con respecto al ICC tanto los hombres como mujeres del área urbana presentaron valores promedios de ICC mayores $(0,95 \pm 0,1$ y $0,9 \pm 0,06$ para hombres y mujeres respectivamente) que los del área rural $(0,93 \pm 0,06$ y $0,88 \pm 0,06$ para hombres y mujeres, respectivamente). La mayor parte de la población estudiada eran personas no fumadoras (Tabla 1 ). 
Tabla 1. Edad, datos antropométricos y presión arterial según población y género, Yantaló julio 2014

\begin{tabular}{|c|c|c|c|c|}
\hline & \multicolumn{2}{|c|}{ Urbano } & \multicolumn{2}{|c|}{ Rural } \\
\hline & $\begin{array}{l}\text { Hombres } \\
\text { media } \pm \mathrm{DE}\end{array}$ & $\begin{array}{c}\text { Mujeres } \\
\text { media } \pm \mathrm{DE}\end{array}$ & $\begin{array}{l}\text { Hombres } \\
\text { media } \pm \mathrm{DE}\end{array}$ & $\begin{array}{c}\text { Mujeres } \\
\text { media } \pm \mathrm{DE}\end{array}$ \\
\hline N (\%) & $62(40,8 \%)$ & $90(59,2 \%)$ & $53(45,7 \%)$ & $63(54,3 \%)$ \\
\hline Edad (años) & $51,24 \pm 13,07$ & $49,59 \pm 13,30$ & $48,43 \pm 11,15$ & $46,79 \pm 11,60$ \\
\hline Peso (kg) & $65,90 \pm 10,96$ & $60,79 \pm 10,42$ & $67,71 \pm 8,60$ & $58,93 \pm 9,67$ \\
\hline Talla (cm) & $161,30 \pm 6,64$ & $150,10 \pm 5,30$ & $161,60 \pm 5,69$ & $147,80 \pm 5,45$ \\
\hline IMC (kg/m2) & $25,24 \pm 3,47$ & $26,95 \pm 4,1$ & $25,90 \pm 2,74$ & $26,93 \pm 4,1$ \\
\hline Cintura (cm) & $87,37 \pm 9,36$ & $88,34 \pm 9,65$ & $89,37 \pm 8,10$ & $86,07 \pm 10,14$ \\
\hline Cadera $(\mathrm{cm})$ & $92,39 \pm 8,29$ & $98,15 \pm 7,84$ & $96,21 \pm 5,06$ & $97,11 \pm 8,19$ \\
\hline \multicolumn{5}{|l|}{ ICC } \\
\hline $\begin{array}{l}\text { Presión Arterial } \\
\text { Sistólica (mmHg) }\end{array}$ & $123,60 \pm 14,75$ & $121,60 \pm 20,53$ & $116,30 \pm 9,66$ & $116,50 \pm 18,82$ \\
\hline $\begin{array}{l}\text { Presión Arterial } \\
\text { Diastólica (mmHg) }\end{array}$ & $74,69 \pm 7,55$ & $70,67 \pm 10,39$ & $71,91 \pm 8,48$ & $69,92 \pm 10,47$ \\
\hline \multicolumn{5}{|l|}{ Tabaquismo (\%) } \\
\hline Fumador & $14(22,6 \%)$ & $0(0 \%)$ & $14(26,4 \%)$ & $0(0 \%)$ \\
\hline No fumador & $34(54,8 \%)$ & $83(92,2 \%)$ & $34(64,2 \%)$ & $57(90,5 \%)$ \\
\hline
\end{tabular}

En la población urbana la prevalencia de HTA fue mayor que la población rural (18,4 y 6\%, respectivamente) con una diferencia significativa $(p=0,0031)$. Con respecto a DM, ICC aumentado, estado nutricional y tabaquismo presentaron valores porcentuales similares entre ambas poblaciones (Tabla 2 ).

Tabla 2. Comparación urbano-rural de los factores de riesgo cardiovascular de Yantaló, julio 2014

\begin{tabular}{|c|c|c|c|c|}
\hline & Urbano (\%) & Rural (\%) & $\mathbf{x}+\dagger$ & p valor \\
\hline \multicolumn{5}{|c|}{ Hipertensión Arterial } \\
\hline Sí & $28(18,4 \%)$ & $7(6 \%)$ & \multirow{2}{*}{8,890} & \multirow{2}{*}{$0,0031^{*}$} \\
\hline No & $124(81 \%)$ & $109(94 \%)$ & & \\
\hline \multicolumn{5}{|c|}{ Diabetes Mellitus } \\
\hline Sí & $9(5,9 \%)$ & $3(2,6 \%)$ & \multirow{2}{*}{1,711} & \multirow{2}{*}{0,1909} \\
\hline No & $143(94,1 \%)$ & $113(97,4 \%)$ & & \\
\hline \multicolumn{5}{|l|}{ Tabaquismo } \\
\hline Sí & $23(15,1 \%)$ & $20(17,3 \%)$ & \multirow{2}{*}{0,2174} & \multirow{2}{*}{0,6410} \\
\hline No & $129(84,9 \%)$ & $96(82,7 \%)$ & & \\
\hline \multicolumn{5}{|c|}{ Estado Nutricional } \\
\hline Bajo peso & $2(1,3 \%)$ & $0(0 \%)$ & \multirow{4}{*}{1,565} & \multirow{4}{*}{0,667} \\
\hline Normal & $49(32,2 \%)$ & $39(33,6 \%)$ & & \\
\hline Sobrepeso & $72(47,4 \%)$ & $55(47,4 \%)$ & & \\
\hline Obesidad & $29(19,1 \%)$ & $22(19 \%)$ & & \\
\hline \multicolumn{5}{|c|}{ Índice Cintura/Cadera } \\
\hline \multicolumn{5}{|l|}{ Varones } \\
\hline Normal & $18(29 \%)$ & $17(32,1 \%)$ & \multirow{2}{*}{0,1250} & \multirow{2}{*}{0,7237} \\
\hline Aumentado & $44(71 \%)$ & $36(67,9 \%)$ & & \\
\hline \multicolumn{5}{|l|}{ Mujeres } \\
\hline Normal & $13(14,4 \%)$ & $17(27 \%)$ & \multirow{2}{*}{3,697} & \multirow{2}{*}{0,0545} \\
\hline Aumentado & $77(85,6 \%)$ & $46(73 \%)$ & & \\
\hline
\end{tabular}

*Prueba exacta de Fisher.

†X2: valor del chi-cuadrado 
Yordan Martínez-Espichán, Rommel Zambrano-Huailla, Alexander Zambrano-Huailla, Anibal Manrique-Acha, Jean Carlo Mayta-Calderón, Alejandra Cardenas-Rojas, Julissa Mogollón-Lavi, Esthefany Seminario-Azula, Stefano Giannoni-Luza, Gabriela Gonzales-Pariona,

\section{DISCUSIÓN}

El estudio reveló que los factores de riesgo cardiovascular se encuentran presentes tanto en la población rural y urbana de Yantaló. De los cinco factores estudiados, sólo la HTA fue la que mostró una diferencia significativa con respecto al lugar de residencia, siendo mayor en el área urbana. Sin embargo los otros factores de riesgo estudiados muestran que el lugar de residencia no influye en la aparición de estos factores.

La población urbana de Yantaló presentó una prevalencia de HTA menor en comparación a otras poblaciones urbanas, como lo reporta Martín et al ${ }^{(13)}$ en Argentina y Zarbato et al $^{(14)}$ en Brasil. Por otro lado, la población rural mostró una prevalencia inferior a lo encontrado en poblaciones rurales de Chile según Navarrete et al ${ }^{(15)}$ y Argentina según De all et al ${ }^{(16)}$. Al comparar la prevalencia de HTA entre ambas poblaciones de Yantaló, se encontró que la HTA fue significativamente mayor en la población urbana con respecto a la población rural. Este hallazgo coincide con otros estudios, realizados por Campos-Noato et al ${ }^{(17)}$ en México y por Miranda et al ${ }^{18)}$ en Perú. Como se sabe, esta prevalencia de HTA se debe a las diferencias en el estilo de vida propias de la población urbana, por ejemplo cambios en la dieta alimentaria y reducción de la actividad física, siendo esta interacción ambiental más el genotipo poblacional, el desencadenante en la aparición de HTA.

La prevalencia de DM en la población urbana fue inferior a lo encontrado por Aschner ${ }^{(19)}$ en Colombia y por Rodrigues et $\mathrm{al}^{\left({ }^{(20)}\right.}$ en Brasil. En la población rural, la prevalencia fue mayor a lo reportado por Aschner ${ }^{(19)}$ en Colombia. Sin embargo, al comparar los hallazgos de ambas poblaciones, no se observaron diferencias entre ambos valores porcentuales. Este resultado obtenido en Yantaló, puede deberse al efecto de la urbanización sobre la población rural, especialmente sobre los estilos de vida, lo que explicaría la similitud de la prevalencia de esta entidad entre ambas poblaciones.

La población urbana de Yantaló presentó una prevalencia de ICC aumentado, bastante mayor que la reportada por Aschner ${ }^{(21)}$ en Colombia. Con respecto a la población rural, la proporción del ICC aumentado fue superior a lo hallado por Arroyo et al ${ }^{(22)}$ en México. Con respecto al estado nutricional, la prevalencia de obesidad fue similar entre la población urbana y rural, resultado que se asemeja a lo realizado por Arroyo et al $^{(22)}$. Esto refleja un desbalance nutricional que presentan ambas poblaciones. Del mismo modo a lo estudiado sobre la DM, cambios en el estilo de vida en el contexto de la urbanización, como por ejemplo reducción de la actividad física y un mayor consumo energético, sería la posible causa de dicho desbalance.

El tabaquismo reportado en la población rural de Yantaló fue superior a lo presentado por Miranda et al ${ }^{(18)}$ en Ayacucho y por Jaganath et $\mathrm{al}^{(23)}$ en Puno. Con respecto a la población urbana, la prevalencia encontrada fue superior a lo encontrado por Jaganath et al ${ }^{(23)}$ en Puno y Lima. Sin embargo, la prevalencia de tabaquismo fue similar para ambas poblaciones de Yantaló. Este resultado, junto a los ya comentados anteriormente, corrobora que la población rural de Yantaló está adquiriendo hábitos de una población urbana.

Se encontraron algunas limitantes en el estudio, como el muestreo de tipo incidental que se utilizó en la zona rural así como la variedad metodológica de las investigaciones citadas, especialmente los rangos de edad que fueron abarcados en los estudios anteriores. A pesar de esto, el presente estudio abarca en su mayoría las edades comprendidas por los estudios citados. Otra limitante fue el no confirmar, mediante pruebas de laboratorio, la presencia de DM en la población. Por otra parte, a nivel metodológico, no se determinó de forma sistemática la conducta alimentaria de ambas poblaciones, así como también la actividad física, datos que hubieran permitido explicar, de manera precisa, nuestros resultados.

En conclusión, el único factor de riesgo que mostró asociación significativa con el lugar de residencia fue la HTA. En contraste, los otros factores de riesgo del estudio no presentaron diferencias entre ambas poblaciones, lo que demuestra que el distrito de Yantaló se encuentra inmerso en un proceso de transición epidemiológica debido a la urbanización.

Agradecimiento: Al Doctor Luis Vásquez, presidente de la Fundación Yantaló, por su asistencia técnica durante el presente trabajo.

\section{REFERENCIAS BIBLIOGRÁFICAS}

1. Segura-Vega L, Agusti R, Parodi-Ramirez J. Factores de riesgo de las enfermedades cardiovasculares en el Perú. Rev Peru Cardiol. 2006;32(2):82-128.

2. OMS. Enfermedades cardiovasculares [Internet]. World Health Organization; 2015 [cited 2015 Nov 15]. Available from: http://www.who.int/mediacentre/factsheets/fs317/es/

3. Ruiz E, Segura L, Agusti R. Uso del Score de Framingham como las indicador de los factores de riesgo de las enfermedades cardiovasculares en la población peruana. Rev Peru Cardiol. 2012;38(3):1-19.

4. Municipalidad distrital de Yantaló. Perfil del proyecto de inversión pública (PIP) mejoramiento y ampliación de la gestión integral de residuos sólidos municipales de la zona urbana de Yantaló, Provincia de Moyobamba, Región San Martin. 2011.

5. Municipalidad Provincial de Moyobamba. Plan de Desarrollo Institucional: PDI 2011-2014. 2011.

6. INEI. Perú: Estimaciones y Proyecciones de Población Económicamente Activa, Urbana y Rural por Sexo y Grupos de Edad, según departamento, 2000-2015. 2010.

7. Kannel W, Wolf P, Verter J, McNamara P. Epidemiologic Assessment of the Role of Blood Pressure in Stroke: the Framingham Study. JAMA.1970; 1970;214(2):301-10

8. Chobanian A, Bakris G, Black H, Cushman W, Green L, Izzo $J$, et al. Seventh report of the Joint National Committee on Prevention, Detection, Evaluation, and Treatment of High Blood Pressure. Hypertension. 2003;42(6):1206-52.

9. Weber M, Schiffrin E, White W, Mann S, Lindholm L, Kenerson $\mathrm{J}$, et al. Clinical Practice Guidelines for the Management 
of Hypertension in the Community. J Clin Hypertens. 2014;16(1):14-26.

10. OMS. Obesidad y sobrepeso [Internet]. World Health Organization; 2015 [cited 2015 Nov 15]. Available from: http://www.who.int/mediacentre/factsheets/fs311/es/

11. World Health Organization. Waist Circumference and WaistHip Ratio. World Health Organization. 2008.

12. Colegio Médico del Perú. Guía nacional de abordaje técnico al tabaquismo. 2010.

13. Marin M, Fábregues G, Rodríguez P. Diaz M, Paez O, Alfie J, et al. Registro Nacional de Hipertensión Arterial: Conocimiento, tratamiento y control de la hipertensión arterial. Estudio RENATA. Rev Argent Cardiol. 2012;80(2):121-9.

14. Zarbato G, Das J, Martins V, Aurélio M. Prevalence of high blood pressure levels and associated factors among adults in Southern Brazil. Arq Bras Cardiol. 2009;93(4):360-94.

15. Navarrete $C$, Cartes-Velásquez R. Prevalencia de hipertensión arterial en comunidades Pehuenches, Alto BioBio. Rev Chil Cardiol. 2012;31(2): $102-7$

16. De All J, Lanfranconi M, Bledel I, Doval H, Hughes A, Laroti A, et al. Prevalencia de la hipertensión arterial en poblaciones rurales del norte argentino. Hipertens y Riesgo Vasc. 2012;29(2):31-5.

17. Campos-Nonato I, Hernández-Barrera L, Rojas-Martínez R, Pedroza A, Medina-García C, Barquera-cerveza S. Hipertensión arterial: Prevalencia, diagnóstico oportuno, control y tendencias en adultos mexicanos. Salud Publica Mex. 2013;55(2):144-50.

18. Miranda J, Gilman R, Smeeth L. Differences in cardiovascular risk factors in rural, urban and rural-to-urban migrants in Peru. Heart. 2011;97(10):787-96.

19. Aschner P. Epidemiología de la diabetes en Colombia. Av en Diabetol. 2010;26(2):95-100.

20. Rodrigues W, Gaban S, Pontes E, Souza C, Gimenes L, Lacerda $P$, et al. Diabetes mellitus and impaired glucose tolerance in urban adult population. Rev Assoc Med Bras (1992). 2014;60(2):118-24.
21. Aschner P. Síndrome metabólico en una población rural y una población urbana de la región andina colombiana. Rev Med. 2007; 15(2):154-62.

22. Arroyo P, Fernández V, Loría A, Pardío J, Laviada H, VargasAncona L, et al. Obesidad, morfología corporal y presión arterial en grupos urbanos y rurales de Yucatán. Salud Publica Mex. 2007;49(4):274-85.

23. Jaganath D, Miranda J, Gilman R, Wise R, Diette G, Miele C, et al. Prevalence of chronic obstructive pulmonary disease and variation in risk factors across four geographically diverse resource-limited settings in Peru. Respir Res. 2015;16(1):40.

Fuentes de financiamiento:

Este artículo ha sido financiado por los autores.

\section{Conflictos de interés:}

Los autores declaran no tener ningún conflicto de interés.

\section{Correspondencia:}

Rommel Eiger Zambrano Huailla

Dirección: Calle Valencia 172, Urb. La Capilla, La Molina. Lima, Perú.

Teléfono: 956302107

Correo electrónico: rommel_334@hotmail.com

Recibido: 29 de setiembre de 2016 Aprobado: 27 de enero de 2017 